\title{
SMALL REPRESENTATIONS \\ OF FINITE DISTRIBUTIVE LATTICES AS CONGRUENCE LATTICES
}

\author{
GEORGE GRÄTZER, IVAN RIVAL, AND NEJIB ZAGUIA
}

(Communicated by Lance W. Small)

\begin{abstract}
A recent result of G. Grätzer, H. Lakser, and E. T. Schmidt states that for any distributive lattice $D$ with $n$ join-irreducible elements, there exists a lattice $L$ with $O\left(n^{2}\right)$ elements, whose congruence lattice $\operatorname{Con} L$ is isomorphic to $D$. We show that this result is best possible.
\end{abstract}

\section{INTRODUCTION}

It is a classical result of R. P. Dilworth (circa 1940)-first published in 1962 in G. Grätzer and E. T. Schmidt [5] - that every finite distributive lattice is the congruence lattice of a lattice. (See G. Grätzer [2] for a brief review of the field (about 40 papers) that grew out of this result.)

In view of the modern interest in algorithmic complexity, attention has turned, in recent years, to computing the minimum size of the lattice $L$ whose congruence lattice $\operatorname{Con} L$ is isomorphic to the given distributive lattice $D$.

It is natural to measure the size of the lattice $L$ in terms of the number of join-irreducible elements of the distributive lattice $D$. For an arbitrary finite distributive lattice $D$ with $n$ join-irreducible elements, it was shown by G. Grätzer and E. T. Schmidt [5] that there is a lattice $L$ of size $O\left(2^{2 n}\right)$ such that Con $L \cong D$. Recently, G. Grätzer and H. Lakser [3] elaborated a construction in which the size of $L$ was reduced to $O\left(n^{3}\right)$. Subsequently, G. Grätzer, H. Lakser, and E. T. Schmidt [4] have announced that they can construct such a lattice with $O\left(n^{2}\right)$ elements. The purpose of this note is to show that no one can do better.

Theorem. Let $\alpha$ be a real number satisfying the following condition: Every distributive lattice $D$ with $n$ join-irreducible elements can be represented as the congruence lattice of a lattice $L$ with $O\left(n^{\alpha}\right)$ elements. Then $\alpha \geq 2$.

Received by the editors July 1, 1993.

1991 Mathematics Subject Classification. Primary 06B10; Secondary 06D05.

Key words and phrases. Congruence lattice, finite lattice, distributive lattice.

The research of all three authors was supported by the NSERC of Canada. 


\section{TWO LEMMAS}

In this section we prove two general lemmas.

If $\Phi$ is a join-irreducible congruence of a finite lattice $L$, then we can represent it in the form $\Phi=\boldsymbol{\Theta}(v, u)$, where $v, u \in L$ and $v \prec u$ (that is, $v$ is covered by $u$ ); as usual, $\Theta(v, u)$ denotes the smallest congruence collapsing $v$ and $u$.

Lemma 1. Let $L$ be a lattice, and let $v_{i}, u_{i} \in L$ satisfy $v_{i} \prec u_{i}$, for $i=1$, 2. Let $\Phi_{i}=\boldsymbol{\theta}\left(v_{i}, u_{i}\right)$, for $i=1,2$. If $\Phi_{1} \prec \Phi_{2}$ in Con $L$, then there is $a$ three-element chain $\left\{e_{1}, h, e_{2}\right\}$ in $L$ such that $\Phi_{i}=\Theta\left(h, e_{i}\right)$, for $i=1,2$, and $e_{1}<h<e_{2}$ or $e_{2}<h<e_{1}$.

Proof. We assume that the reader is familiar with the basic concepts and notations of projectivity in lattices (see, for instance, pages 129-130 of [1]). Since $v_{1} \equiv u_{1}\left(\boldsymbol{\Theta}\left(v_{2}, u_{2}\right)\right)$ and $v_{1} \prec u_{1}$, by Theorem III.1.2 ibid, there is a sequence of projectivities

$$
u_{2} / v_{2}=y_{1} / x_{1} \nearrow y_{2} / x_{2} \searrow y_{3} / x_{3} \nearrow \ldots \searrow y_{n} / x_{n}=u_{1} / v_{1},
$$

for some natural number $n>1$. Obviously,

$$
\boldsymbol{\Phi}_{2}=\boldsymbol{\Theta}\left(x_{1}, y_{1}\right) \geq \boldsymbol{\Theta}\left(x_{2}, y_{2}\right) \geq \boldsymbol{\Theta}\left(x_{3}, y_{3}\right) \geq \cdots \geq \boldsymbol{\Theta}\left(x_{n}, y_{n}\right)=\Phi_{1} \text {. }
$$

Since $\Phi_{2} \succ \Phi_{1}$, there is an $i$ satisfying $\boldsymbol{\Theta}\left(x_{i}, y_{i}\right)=\Phi_{2}, \boldsymbol{\Theta}\left(x_{i+1}, y_{i+1}\right)=\Phi_{1}$, and $1 \leq i<n$. For this $i$, either $y_{i} / x_{i} \nearrow y_{i+1} / x_{i+1}$ or $y_{i} / x_{i} \searrow y_{i+1} / x_{i+1}$. In the first case, set $h=y_{i} \wedge x_{i+1}, e_{2}=x_{i}$, and $e_{1}=y_{i}$; obviously, $e_{1}<h<$ $e_{2}, \Phi_{1}=\boldsymbol{\Theta}\left(h, e_{1}\right)$, and $\boldsymbol{\Phi}_{2}=\boldsymbol{\Theta}\left(h, e_{2}\right)$. If $y_{i} / x_{i} \searrow y_{i+1} / x_{i+1}$, we proceed similarly, and obtain $\Phi_{1}$ on top.

The second lemma deals with join-independence. A set $A$ in a lattice $L$ is join-independent if for any $a \in A$ and finite subset $A_{1} \subseteq A$, the inequality $a \leq \bigvee A$ implies that $a \in A_{1}$.

Lemma 2. Let $L$ be a lattice, let $A \subseteq L$, and let $b \in L$ be a lower bound of $A$ in $L$. If $\{\boldsymbol{\theta}(b, x) \mid x \in A\}$ is join-independent in $\operatorname{Con} L$, then $A$ is join-independent in $L$.

Proof. Indeed, if $a \leq \bigvee A_{1}$ for some finite $A_{1} \subseteq A$, then

$$
\boldsymbol{\Theta}(b, a) \leq \bigvee\left\{\boldsymbol{\Theta}(b, x) \mid x \in A_{1}\right\}
$$

a contradiction.

Finally, observe that in a distributive lattice, a set $A$ of join-irreducible elements is independent iff the elements are pairwise incomparable. So we obtain the following

Corollary. Let $L$ be a lattice, let $A \subseteq L$, and let $b \in L$ be a lower bound of $A$ in $L$. If $\{\boldsymbol{\Theta}(b, x) \mid x \in A\}$ is a set of pairwise incomparable join-irreducible congruences, then $A$ is join-independent in $L$.

\section{Proof of The Theorem}

Let $n$ be a natural number. Let $D_{n}$ be a distributive lattice whose partially ordered set $P_{n}$ of join-irreducible elements is bipartite (that is, every element is 
either maximal or minimal) and in which there are $O\left(n^{2}\right)$ covering edges. Let $L_{n}$ be a lattice satisfying Con $L_{n} \cong D_{n}$, and contrary to the Theorem assume that $L_{n}$ has $O\left(n^{\alpha}\right)$ elements where $\alpha<2$.

There are $O\left(n^{2}\right)$ covering pairs of join-irreducible congruences of $L_{n}$. By Lemma 1, each pair $\Phi_{1} \prec \Phi_{2}$ corresponds to a three-element chain $e_{1}<h<e_{2}$. Since there are $O\left(n^{2}\right)$ covering pairs of join-irreducible congruences and there are $O\left(n^{\alpha}\right)$ elements in $L_{n}$, there must be an element $h \in L_{n}$ that appears as the middle element of $O\left(\frac{n^{2}}{n^{\alpha}}\right)=O\left(n^{2-\alpha}\right)$ three-element chains. For half of these chains, still $O\left(n^{2-\alpha}\right)$ in number, the top interval (or dually, the bottom interval) defines the larger congruence. So we obtain in $L_{n}$ an element $h$ and a set $A$ of elements, $O\left(n^{2-\alpha}\right)$ in number, so that all the $\Theta(h, x)$ are maximal (or all are minimal) join-irreducible congruences of $L_{n}$. Obviously, these congruences are pairwise incomparable. By the Corollary to Lemma 2, $A$ - under joingenerates a free join-semilattice $F$ (or in the dual case, a free meet-semilattice). The set $F \subseteq L_{n}$ has $O\left(2^{n^{2-\alpha}}\right)$ elements. But this is a contradiction since $L_{n}$ has only $O\left(n^{2}\right)$ elements.

\section{REFERENCES}

[1] G. Grätzer, General lattice theory, Pure Appl. Math. Ser., Academic Press, New York, 1978; Mathematische Reihe, Band 52, Birkhäuser Verlag, Basel; Akademie Verlag, Berlin.

[2] _ Results on the congruence lattice of a lattice, The Dilworth Theorems. Selected Papers of Robert P. Dilworth (K. P. Bogart, R. Freese, and J. P. S. Kung, eds.), Birkhäuser Verlag, Basel-Boston, 1990, pp. 460-464.

[3] G. Grätzer and H. Lakser, Congruence lattices of planar lattices, Acta Math. Hungar. 60 (1992), 251-268.

[4] G. Grätzer, H. Lakser, and E. T. Schmidt, Congruence lattices of small planar lattices. Proc. Amer. Math. Soc. (to appear).

[5] G. Grätzer and E. T. Schmidt, On congruence lattices of lattices, Acta Math. Acad. Sci. Hungar. 13 (1962), 179-185.

(G. Grätzer) Department of Mathematics, University of Manitoba, Winnipeg, ManiTOBa, CANADa R3T 2N2

E-mail address: George_Gratzer@umanitoba.ca

(I. Rival and M. Zaguia) Department of Computer Science, University of Ottawa, Ottawa, Ontario, Canada K1N 6N5

E-mail address: rival@csi.uottawa.ca

E-mail address: zaguia@csi.uottawa.ca 